\title{
ACMT Position Statement: Medication Administration and Safety During the Response to COVID-19 Pandemic
}

\author{
Brenna M. Farmer ${ }^{1} \cdot$ Jon B. Cole ${ }^{2} \cdot$ Travis D. Olives $^{3} \cdot$ Natalija M. Farrell $^{4,5} \cdot$ Rama Rao $^{6} \cdot$ Lewis S. Nelson $^{7}$. \\ Maryann Mazer-Amirshahi ${ }^{8} \cdot$ Andrew I. Stolbach $^{9}$
}

Received: 4 June 2020 / Revised: 10 June 2020 / Accepted: 11 June 2020 / Published online: 3 July 2020

(C) American College of Medical Toxicology 2020

Keywords Toxicology · COVID-19 · Coronavirus · Pharmacy $\cdot$ Medication safety

The position of the American College of Medical Toxicology (ACMT) is as follows:

The coronavirus disease 2019 (COVID-19) pandemic has created medication safety challenges affecting patients and staff. ACMT recommends modification of some health care delivery practices to provide effective care while responding to drug shortages, infection risk, and staffing changes. All practices must also consider staff safety and preservation of personal protective equipment (PPE). We encourage collaboration of physicians and clinical pharmacists at every phase of design and implementation of medication policy changes.

Supervising Editor: Mark B. Mycyk, MD

Andrew I. Stolbach

positionstatements@acmt.net

1 Department of Emergency Medicine, Weill Cornell Medicine, New York, NY, USA

2 Department of Emergency Medicine, Hennepin Healthcare, Minneapolis, USA

3 Minnesota Poison Control System, Department of Emergency Medicine, Hennepin Healthcare, Minneapolis, MN, USA

4 Department of Pharmacy, Boston Medical Center, Boston, MA, USA

\section{Background}

The COVID-19 pandemic requires new approaches to delivery of health care. The combination of large numbers of ventilated patients, shortages of common medications, infection risks to health care workers, and lack of effective drug treatments has created safety challenges related to drug administration.

This statement by the American College of Medical Toxicology is intended to provide guidance for safe medication administration and protection of health care team members in the face of the COVID-19 pandemic. Collaboration

5 Department of Emergency Medicine, Boston University School of Medicine, Boston, MA, USA

6 Weill Cornell Medicine-New York Presbyterian Hospital, New York, NY, USA

7 Rutgers New Jersey Medical School, Newark, NJ, USA

8 Department of Emergency Medicine, MedStar Washington Hospital Center and Georgetown University School of Medicine, Washington, DC, USA

9 Johns Hopkins University School of Medicine, Baltimore, MD, USA 
between physicians (including medical toxicologists) and other members of the care team in implementation of these policies is essential to optimize patient care and staff safety during this unprecedented time [1].

\section{Staff Safety}

Health care workers face a risk of occupational transmission of disease that is complicated by hospital PPE shortages [2]. Patient care requires direct contact, but every encounter increases the opportunity for viral transmission and consumes PPE, which is in limited supply. Procedures that potentially generate aerosols, such as endotracheal intubation or administration of nebulized medications, are often necessary but may increase risk of viral transmission [3]. We recommend medication administration practices that minimize risk of medical staff infection.

\section{Recommendations}

1. To reduce PPE use and minimize risk of staff infection, bundle medication administration, and phlebotomy times when possible.

2. Use medications that require less frequent dosing when this can be done safely and effectively.

3. Use long extension tubing to allow for infusion pumps to be placed outside the patient room, which will limit staff exposure and conserve PPE.

4. Nebulized medications should only be used in negative pressure rooms and airborne infection isolation. Substitute nebulized medications with metered dose inhalers (with spacer) whenever possible. (The data to support this risk are limited, but we recommend a conservative approach until more is known.)

\section{Education}

Increased demand for generic injectables, especially sedatives, analgesics, and paralytics for ventilated patients, has resulted in drug shortages, forcing the adoption of different formulations of first-line medications and use of second- and third-line medications [4-6]. Because our understanding of COVID-19 is constantly growing in the face of new information, treatment protocols are continuously evolving. Hospitals have adopted - and in many cases discontinued - protocols for use of unproven medications such as hydroxychloroquine and azithromycin.

At the same time that new or alternative therapies are being introduced, many hospital workers are staffing unfamiliar units or have had their scope of practice expanded due to redeployment. Hospitals have needed to suspend non-emergent procedures and shift ambulatory services to telemedicine while simultaneously opening inpatient COVID-specific and medical intensive care units. Hospitals also face staffing shortages caused by worker illness. By necessity, hospitals have had to recruit temporary employees and redeploy workers from underused units to staff new units and replace sick health care workers.

\section{Recommendations}

1. Ensure that new workers are educated on commonly used medications and administration procedures. Educate providers on the use of new medications or unfamiliar formulations. Use information technology to provide clinical decision support in the electronic health record. Ideally, computerized order entry and barcoding should already be updated at the time staff is directed to substitute medications.

2. Updates to treatment protocols based on new evidence should be communicated to staff in a timely manner. Additionally, these changes can be added to clinical decision support tools and electronic ordering platforms.

\section{Research}

Currently, there are no proven direct COVID-19 therapies. There are several regulatory mechanisms for administration of investigational medications. Off-label administration - the use of an approved medication for an unapproved use-has the least regulatory oversight and requirement for structured data collection. Chloroquine, hydroxychloroquine, and azithromycin have been used in this manner with mixed but generally negative results. FDA expanded access programs and emergency use authorizations (EUA) ensure more protocolized administration of drugs to patients without good existing therapeutic options [7, 8]. An EUA, granted by the FDA in event of a public health emergency, allows for administration of an unapproved drug. Expanded access is a mechanism for drug administration granted by the FDA with cooperation of the manufacturer and local IRB. Expanded access and EUA allow for collection of structured data, but not rigorous comparison of drugs. The efficacy and safety of investigational medications can best be ascertained through controlled clinical trials [9]. Clinical trials offer regulatory and ethical oversight while providing a way to understand better means of disease treatment. 


\section{Recommendations}

1. When possible, administer investigational medications for treatment as part of controlled clinical trials.

2. Use expanded access or off-label administration only as a last resort for administration of investigational drugs.

3. Report adverse drug events that may occur as part of clinical trials to the appropriate institutional committees and FDA's MedWatch.

\section{Compliance with Ethical Standards}

\section{Conflicts of Interest None \\ Sources of Funding None}

Disclaimer While individual practices may differ, this is the position of the American College of Medical Toxicology at the time written, after a review of the issue and pertinent literature.

\section{References}

1. Farmer BM, Hayes BD, Rao R, Farrell N, Nelson L. The role of clinical pharmacists in the emergency department. J Med Toxicol. 2018;14(1):114-6.

2. Zhang Z, Liu S, Xiang M, Li S, Zhao D, Huang C, et al. Protecting healthcare personnel from 2019-nCoV infection risks: lessons and suggestions. Front Med [Internet]. 2020 Available from: https://doi. org/10.1007/s11684-020-0765-x.

3. Tran K, Cimon K, Severn M, Pessoa-Silva CL, Conly J. Aerosol generating procedures and risk of transmission of acute respiratory infections to healthcare workers: a systematic review. PLoS One. 2012; 7:e35797.

4. Verity R, Okell LC, Dorigatti I, Winskill P, Whittaker C, Imai N, et al. Estimates of the severity of coronavirus disease 2019: a modelbased analysis. Lancet Infect Dis [Internet]. 2020 Available from: https://doi.org/10.1016/S1473-3099(20)30243-7.

5. American Society of Health-System Pharmacists (ASHP). Current Drug Shortages [Internet]. ASHP Drug Shortage List: [cited 2020 Apr 7]. Available from: https://www.ashp.org/Drug-Shortages/ Current-Shortages

6. Alexander GC, Qato DM. Ensuring access to medications in the US during the COVID-19 pandemic. JAMA [Internet]. 2020 Available from: https://doi.org/10.1001/jama.2020.6016.

7. Jarow JP, Lurie P, Crowley Ikenberry S, Lemery S. Overview of FDA's expanded access program for investigational drugs. Ther Innov Regul Sci. 2017;51:177-9.

8. US Food and Drug Administration. Emergency use authorization of medical products and related authorities: guidance for industry and other stakeholders. https://www.fda.gov/regulatoryinformation/ search-fda-guidance-documents/emergency-use-authorizationmedical-products-andrelated-authorities\#euas. Accessed May 17, 2020

9. ACMT position statement on off-label prescribing during COVID19 pandemic. https://www.acmt.net/_Library/Positions/ACMT_ Position_Statement_on_Off-Label_Prescribing_during_COVID19_Pandemic_5-3-20.pdf Accessed May 17, 2020.

Publisher's Note Springer Nature remains neutral with regard to jurisdictional claims in published maps and institutional affiliations. 